\title{
High levels of gut carriage of antimicrobial-resistant Escherichia coli in community settings in Rio de Janeiro, Brazil
}

\author{
Káris Maria de Pinho Rodrigues ${ }^{1} \cdot$ Danielle Ferreira de Rezende ${ }^{2} \cdot$ Michelle Pessanha Pinto $^{1}$. \\ Samantha dos Santos Tufic-Garutti ${ }^{2}$. João Vitor Almeida Ramalho ${ }^{1} \cdot$ Luís Guilherme de Araújo Longo $^{2}$. \\ Valéria Brígido de Carvalho Girão ${ }^{2} \cdot$ Gabriela Caramano de Oliveira $^{2} \cdot$ Lucas Cecílio Vilar $^{2}$. \\ Beatriz Meurer Moreira ${ }^{2,3}$ (B)
}

Received: 19 July 2021 / Accepted: 24 December 2021 / Published online: 6 January 2022

(c) Sociedade Brasileira de Microbiologia 2022

\begin{abstract}
The prevalence and risk factors for gut carriage of antimicrobial-resistant Escherichia coli among individuals living in the community in Rio de Janeiro, Brazil, are unknown. The aim of this study was to determine the prevalence of colonization with antimicrobial-resistant $E$. coli, including isolates producing ESBL and harboring plasmid-mediated quinolone resistant (PMQR) genes in this community. We performed a cross-sectional study and analyzed fecal specimens of individuals attending outpatient clinics in the city from January 2015 to July 2019. We investigated susceptibility to antimicrobial agents by disc diffusion tests and used PCR to determine ESBL types, PMQR, and the virulence genes that characterize an isolate as extraintestinal pathogenic E. coli (ExPEC). Among the 623 subjects, $212(34 \%)$ carried an isolate resistant to at least one of the tested antimicrobial agents, with the highest frequencies of resistance to ampicillin (26\%), trimethoprim-sulfamethoxazole (19\%), cefazolin (14\%), and ciprofloxacin (CIP, 9\%). In addition, 13\% (81) of subjects carried a multidrug-resistant- $E$. coli (MDR-E), including 47 (8\% of all isolates) ESBL-producing E. coli (ESBL-E), mainly of CTX-M-8 (15, 32\%) and CTXM-15 (9, 20\%) types. PMQR genes were present in 7\% (42) of all isolates, including 60\% (32) of the 53 resistant to CIP. Previous use of antimicrobial agents, particularly fluoroquinolones, was a risk factor for colonization with MDR-E (25\%, $20 / 81$ vs $13 \%, 70 / 542, p=0.01)$, ESBL-E $(28 \%, 13 / 47$, vs $13 \%, 77 / 576, p=0.01)$, and resistance to CIP $(26 \%, 14 / 53$, vs $12 \%$, $70 / 570, p=0.01)$. The most pathogenic phylogroups B2, C, and D were $37 \%$ of the MDR-E, $30 \%$ of the ESBL-E, $38 \%$ of the CIP-resistant, and $31 \%$ of PMQR gene carrying E. coli isolates. We show that carriage of MDR-E (mostly ESBL-E) reached high levels in the community in Rio de Janeiro, increased by the selection of antimicrobial agents. Much of the resistant $E$. coli isolates are potential pathogenic strains. The widespread use of antimicrobial agents during the COVID-19 pandemic in Brazil may have worsened this picture.
\end{abstract}

Keywords Escherichia coli $\cdot$ Antimicrobial resistance $\cdot$ Carriage $\cdot$ ESBL

Responsible Editor: Afonso Luis Barth

Beatriz Meurer Moreira

bmeurer@micro.ufrj.br

1 Faculdade de Medicina, Universidade Federal Do Rio de Janeiro, Rio de Janeiro, Brazil

2 Instituto de Microbiologia Paulo de Góes, Universidade Federal Do Rio de Janeiro, Rio de Janeiro, Brazil

3 Laboratório de Investigação Em Microbiologia Médica (LIMM), Instituto de Microbiologia Paulo de Góes, Universidade Federal Do Rio de Janeiro, Av. Carlos Chagas Filho, 373; Centro de Ciências da Saúde, Bloco i, Sala I2-59, CEP 21941-902, Rio de Janeiro, RJ, Brazil

\section{Introduction}

Infections by Enterobacterales isolates usually originate in the gut microbiota. The carriage of multidrug-resistant (MDR) isolates, such as extended-spectrum beta-lactamase (ESBL)-producing gram-negative bacteria, is of concern because of the increased risk of resistant infections. Exposure to antimicrobial agents and travel to high-risk geographic regions [1-3], among other factors [4], are associated with high rates of carriage of MDR bacteria.

The increasing prevalence of carriage of ESBL producing Enterobacterales worldwide is particularly worrisome because it may severely limit antimicrobial treatment options 
and increase chances of carbapenem use and healthcare costs $[5,6]$. CTX-M type is the most common among a broad range of ESBL enzymes [7, 8], with CTX-M-15 as the most widespread variant $[9,10]$. Guidelines to control the spread of antimicrobial-resistant pathogens recommend screening for ESBL-producing isolates in high-risk settings, such as during hospital admission of patients previously colonized with MDR bacteria and admission to cancer or intensive care units $[11,12]$.

Escherichia coli is the most frequent commensal microorganism recovered in the human intestinal microbiota [13]. However, genetic traits carried by E. coli isolates differentiate commensal to pathogenic strains [14], such as extraintestinal pathogenic E. coli (ExPEC) and uropathogenic E. coli (UPEC) $[15,16]$. It is expected that, by 2050, infections caused by MDR ExPEC isolates will be responsible for more than three million deaths each year [17]. This outcome will likely be much worse after the current pandemic, because the use of antimicrobial agents should have increased significantly by the (unfound) recommendation of the routine use of antimicrobial agents for COVID-19 patients in certain countries such as Brazil, even for mild or moderate cases [18].

UPEC isolates belong predominantly to phylogenetic groups $\mathrm{B} 2, \mathrm{D} / \mathrm{E}$ or $\mathrm{F}$, and commensal E. coli isolates, to groups B1 or A [19, 20]. Further sub-grouping of UPEC isolates by multilocus sequence typing (MLST) has shown that a limited set of $E$. coli lineages are responsible for a large proportion of extraintestinal infections [21]. Epidemiological studies have shown that lineages ST131, ST73, ST95 and ST69 are the most prevalent ExPEC, especially ST131, the main pandemic clone [22].

In Brazil, MDR E. coli (MDR-E) has already been described in farm animals [23, 24], chicken carcasses [25], wild birds [26], coastal waters [27], and as a cause of infections [28], but not as colonizers among individuals in the community. This information is a useful parameter for empiric antimicrobial therapy and a proxy for the general quality of people's health. Therefore, the present study aimed to investigate the prevalence and risk factors for antimicrobial resistance among $E$. coli gut isolates from individuals living in the community in Rio de Janeiro city, the second biggest city of Brazil.

\section{Materials and methods}

\section{Patient population and bacterial isolates}

From January 2015 to July 2019, we invited outpatients and their companions over 18 years old attending three primary-care medical clinics to participate in the study. Subjects received instructions to self-collect a fecal specimen with an anal swab containing Amies transport media. In our research laboratory, the specimen containing swab tip was transferred to a microtube with $1 \mathrm{~mL}$ skim milk, tryptone, glucose, and glycerin (STGG), vortexed for approximately $30 \mathrm{~s}$, and stored at $-20^{\circ} \mathrm{C}$. The Ethics Committee approved the study (CAAE: 49,843,315.0.0000.5257).

\section{Microbiological methods}

We plated aliquots of specimens containing STGG in three types of culture media as follows. We seeded $10 \mu \mathrm{L}$ aliquots onto plain MacConkey agar (DifcoTM) to guarantee to obtain isolates from every subject, and onto $2 \mu \mathrm{g} / \mathrm{mL}$ ceftriaxone (Agila ${ }^{\circledR}$ ) containing MacConkey agar, to select for ESBL-producing E. coli. In addition, we inoculated a $50 \mu \mathrm{l}$ aliquot of STGG into $9 \mathrm{~mL}$ of trypticase soy broth (TSB) (DifcoTM) containing zinc sulfate $(70 \mu \mathrm{g} / \mathrm{mL})$ and a $10 \mu \mathrm{g}$ ertapenem (ETP) disc to select for carbapenemaseproducing E. coli. Finally, we transferred isolates identified by matrix-assisted laser desorption/ionization-time of flight (MALDI-TOF-MS) (Bruker Daltonics®) as E. coli to a microtube containing $1 \mathrm{~mL}$ skim milk (DifcoTM) and glycerol $(10 \%)$ (Sigma $\left.{ }^{\circledR}\right)$ and stored at $-20^{\circ} \mathrm{C}$. Therefore, we saved a maximum of three isolates (colonies) from each subject. We followed a hierarchical preference to select which isolates to save from each specimen and maximize the chance of detecting antimicrobial resistance: we prioritized colonies grown on MAC agar supplemented with antimicrobial agents. Otherwise, we picked colonies grown on plain MAC agar.

\section{Antimicrobial susceptibility tests}

To assess if we could use a single colony from the primary plate of each subject, we ran a small pilot study. We performed antimicrobial susceptibility tests for 12 drugs, by disc diffusion, with three colonies saved from 20 subjects. In just three subjects, we detected one difference in resistance for one drug among the colonies from the same plate. In none of these three cases, the observed difference would cause misclassification of an isolate as MDR [29]. Therefore, we proceeded with testing one isolate from each participant for susceptibility, by disc diffusion according to CLSI 2018 to the following antimicrobial agents: amikacin (AMI), amoxicillin-clavulanate (AMC), ampicillin (AMP), cefazolin (CFZ), cefepime (CPM), cefotaxime (CTX), cefoxitin (CFO), ceftazidime (CAZ), cefuroxime (CRX), ciprofloxacin (CIP), ertapenem (ETP), fosfomycin (FOS), gentamicin (GEN), nitrofurantoin (NIT), and sulfamethoxazole-trimethoprim (SUT). ESBL production was determined with a disc synergy test [30], using CPM, CAZ, CTX, and AMC discs. We classified isolates as MDR as proposed [31]. Accordingly, all ESBL-producing isolates were classified as MDR. 


\section{Detection of antimicrobial resistance genes}

We screened ESBL-producing isolates for the presence of genes encoding CTX-M, TEM, SHV, and GES type betalactamases. The presence of CTX-M-1, CTX-M-2, CTX-M8, and CTX-M-9 groups and TEM, SHV, and GES genes was detected by multiplex PCR, confirmed in simplex reactions [32], and amplified DNA fragments were sequenced (Macrogen, Seoul, South Korea).

$\mathrm{CFO}$ and $\mathrm{AMC}$ resistant isolates were screened for genes associated with plasmid AmpC (pAmpC); multiplex PCR was performed for genes encoding MOX-like, CMY-1-like, LAT-like, CMY-2-like, DHA-like, ACC-like, MIR, and ACT beta-lactamases [33].

Multiplex PCR was performed for $m c r-1, m c r-2, m c r-3$, $m c r-4$, and $m c r-5$ genes [34]. We confirmed results obtained by multiplex PCR in simplex reactions and sequenced amplified DNA fragments (Macrogen, Seoul, South Korea).

For each of the genes we screened, we used as control an isolate from our laboratory collection with a positive PCR amplicon confirmed by sequencing.

\section{PMQR gene detection}

We investigated the presence of PMQR genes in all isolates by multiplex PCR, as previously described: $q n r D$, $q n r C$, and qnr Vc [35], qnrA, qnrS, qnrB [36], $\operatorname{aac}\left(6^{\prime \prime}\right)-\mathrm{Ib}$ [37], and qepA [38]. For the detection of aac(6")-Ib-cr, purified $\operatorname{aac}\left(6^{\prime \prime}\right)-\mathrm{Ib}$ PCR products were sequenced (Macrogen, Seoul, South Korea).

\section{Phylogenetic groups and ExPEC classification}

We classified all isolates into phylogenetic groups by multiplex PCR [39-41]. Those belonging to phylogenetic groups $\mathrm{B} 2$, D/E, or F were classified as ExPEC based on the presence of two or more defined virulence markers (papAH, $s f a / f o c \mathrm{DE}, a f a / d r a \mathrm{BC}$, iut A, and kpsMT II) [42]. Virulence genes were screened with one multiplex and three simplex PCR tests [43-45].

\section{Statistical analysis}

We described numerical data by their median and interquartile range, and categorical data by absolute counts and percentages. Comparisons of distributions of resistance to specific antimicrobial agents was performed with the chi-square test or Fisher's exact test. Potential statistical associations between the study covariates and the acquisition of MDR-E or ESBL-E were explored in bivariate analyses performed with logistic regression. Statistical significance was defined by a two-sided $p$-value $<0.05$. All analyses were performed with IBM® SPSS v. 23 software.

\section{Results}

We recruited 677 volunteers and excluded 54 (8\%) because no $E$. coli isolates were obtained from fecal specimens. Among the 623 participants included in the study, the median age was 42 years (IQR: $31-56$ ), and $67 \%$ were female. Most participants had concluded secondary or tertiary school levels $(n=508,82 \%)$, and $188(30 \%)$ reported a comorbid condition (including diabetes, hypertension, and autoimmune diseases). A total of 90 (14\%) participants reported the use of antimicrobial agents in the previous 6 months, with the predominance of beta-lactams $(n=54$. $9 \%)$ followed by fluoroquinolones $(n=13.2 \%)$. None had an infectious syndrome at the time of sampling.

\section{Antimicrobial resistance, ESBL production, and MDR isolates}

Among the 623 participants, 212 (34\%) carried an isolate resistant to at least one of the tested drugs. The higher resistance frequencies were for AMP, SUT, and CFZ, as shown in Table 1. Resistance to CIP and the presence of ESBL-E detected by disc synergy tests reached almost $10 \%$ each, and MDR-E was detected in $13 \%$ of study participants (Table 1). Among ESBL-E isolates, $36 \%$ were also resistant to CIP and 30\% harbored PMQR

Table 1 Frequency of antimicrobial resistance among 623 E. coli study isolates

\begin{tabular}{ll}
\hline Antimicrobial agent/resistance phenotype & $\begin{array}{l}\text { Number }(\%) \\
\text { of resistant } \\
\text { isolates }\end{array}$ \\
\hline Ampicillin & $164(26)$ \\
Trimethoprim-sulfamethoxazole & $120(19)$ \\
Cefazolin & $89(14)$ \\
Ciprofloxacin & $53(9)$ \\
Cefotaxime & $52(8)$ \\
Cefuroxime & $46(7)$ \\
Cefepime & $39(6)$ \\
Cefoxitin & $25(4)$ \\
Gentamicin & $19(3)$ \\
Ceftazidime & $18(3)$ \\
Amoxicillin/clavulanate & $15(2)$ \\
Nitrofurantoin & $7(1)$ \\
Fosfomycin & $3(0.5)$ \\
Amikacin & $1(0.2)$ \\
Ertapenem & 0 \\
ESBL & $47(8)$ \\
MDR & $81(13)$ \\
\hline
\end{tabular}

$E S B L$, extended-spectrum beta-lactamase producing isolate; $M D R$, multidrug-resistant isolate 
genes. Considering two study periods (2015-2016 and 2017-2019), carriage of MDR-E increased from $11 \%$ $(39 / 341)$ to $15 \%(42 / 282, p=0.23)$, ESBL-E from $6 \%$ $(20 / 341)$ to $10 \%(27 / 270, p=0.09)$, and resistance to CIP from $6 \%(22 / 319)$ to $11 \%(31 / 251, p=0.06)$.

\section{Presence of PMQR genes}

Among the study isolates, 42 (7\%) carried a PMQR gene, including 17 susceptible to CIP, as shown in Table 2.

Table 2 Distribution of PMQR genes among E. coli isolates according to ciprofloxacin resistance

\begin{tabular}{lllll}
\hline PMQR & \multicolumn{2}{l}{ Number (\%) of isolates } & $p$ \\
\cline { 2 - 4 } & Total $N=623$ & CIP R N=53 (9) & $\begin{array}{l}\text { CIP S } \\
N=570 \\
(91)\end{array}$ & \\
\hline qnrB & $23(4)$ & $9(17)$ & $14(2)$ & $<\mathbf{0 . 0 1}$ \\
qnrS & $9(1)$ & $3(6)$ & $6(1)$ & 0.06 \\
aac(6')-Ib-cr & $7(1)$ & $4(8)$ & $3(0.5)$ & $<\mathbf{0 . 0 1}$ \\
qepA & $2(0.3)$ & $1(2)$ & $1(0.2)$ & 0.3 \\
qnrB + & & & & \\
aac(6')-Ib-cr & $1(0.2)$ & 0 & $1(0.2)$ & 0.9 \\
Total & $42(7)$ & $17(32)$ & $25(4)$ & $<\mathbf{0 . 0 1}$ \\
\hline
\end{tabular}

$P M Q R$, plasmid-mediated quinolone resistance genes; $C I P R$, ciprofloxacin resistant isolates; $C I P S$, ciprofloxacin susceptible isolates. $p$ values $<0.05$ are shown in bold

\section{Bivariate analysis of variables associated with antimicrobial resistance phenotypes and the presence of PMQR genes}

The potential associations of study variables with carriage of MDR-E, ESBL-E, and resistance to CIP are described in Table 3. We found no correlation between age, gender, and education level with any of the three outcomes. Previous antimicrobial use, particularly CIP, was associated with colonization with carriage of MDR-E, ESBL-E, and resistance to CIP $(p=0.01)$.

\section{ESBL types}

Almost all ESBL types were CTX-M, more than half CTX-M8 or CTX-M-15, as shown in Table 4. We did not detect any isolate suspect of carbapenemase-producing, nor $\mathrm{pAmpC}$ or $\mathrm{mcr}$ carrying isolates.

\section{Pathogenic phylogenetic groups and ExPEC}

Among all E. coli isolates, 204 (33\%) belonged to phylogenetic groups B2, D/E, or F, including 37\% of the MDR-E, $31 \%$ of the ESBL producing, $31 \%$ of the PMQR-carrying, and $38 \%$ of the CIP resistant isolates. Of the B2, D/E, or F isolates, 120 (59\%) were classified as ExPEC based on the presence of virulence markers.

Table 3 Bivariate analysis of sociodemographic and clinical characteristics of subjects carrying ESBL-producing or MDR E. coli

\begin{tabular}{|c|c|c|c|c|c|c|c|c|c|}
\hline \multirow[t]{3}{*}{ Variable } & \multicolumn{9}{|c|}{ Number and (\%) of subjects } \\
\hline & \multirow[b]{2}{*}{ Yes $N=81$} & \multicolumn{2}{|l|}{ MDR } & \multirow[b]{2}{*}{ Yes $N=47$} & \multicolumn{2}{|l|}{$\mathrm{ESBL} * *$} & \multicolumn{3}{|l|}{ CIP-R } \\
\hline & & No $N=542$ & $\mathrm{p}$ & & No $N=576$ & $\mathrm{p}$ & Yes $N=53$ & No $\mathrm{N}=570$ & $\mathrm{p}$ \\
\hline Female sex & $49(61)$ & $371(68)$ & 0.16 & $27(57)$ & $393(68)$ & 0.14 & $17(32)$ & $191(34)$ & 0.88 \\
\hline Secondary or tertiary level education & $62(77)$ & $447(82)$ & 0.26 & $36(77)$ & $472(82)$ & 0.46 & $43(81)$ & $465(82)$ & 1.00 \\
\hline Comorbidity present & $12(15)$ & $71(13)$ & 0.71 & $8(17)$ & $75(13)$ & 0.32 & $19(36)$ & $169(30)$ & 0.35 \\
\hline \multicolumn{10}{|l|}{ Study period } \\
\hline 2015-2016 & $39(48)$ & $302(56)$ & 0.23 & $20(43)$ & $321(56)$ & 0.09 & $22(42)$ & $319(56)$ & 0.06 \\
\hline 2017-2019 & $42(52)$ & $240(44)$ & & $27(57)$ & $255(44)$ & & $31(58)$ & $251(44)$ & \\
\hline Used antimicrobial agent & $20(25)$ & $70(13)$ & 0.01 & $13(28)$ & $77(13)$ & 0.02 & $14(26)$ & $70(12)$ & 0.01 \\
\hline \multicolumn{10}{|l|}{ Antimicrobial class } \\
\hline Fluoroquinolone $(N=13)$ & $5(39)$ & $8(6)$ & 0.01 & $4(9)$ & $9(1)$ & 0.01 & $4(8)$ & $9(2)$ & 0.01 \\
\hline Other classes $(N=77)^{*}$ & $15(20)$ & $62(19)$ & & $9(19)$ & $68(12)$ & & $10(18)$ & $67(12)$ & \\
\hline No use & $61(11)$ & $472(75)$ & & $34(72)$ & $499(87)$ & & $39(74)$ & $494(86)$ & \\
\hline
\end{tabular}

$E S B L$, extended-spectrum beta-lactamase producing $E$. coli isolates; $M D R$, multidrug-resistant $E$. coli isolate; $C I P-R$, ciprofloxacin resistant isolates

*54 (70\%) subjects took a betalactam drug; **included among MDR isolates. $p$ values $<0.05$ are shown in bold 
Table 4 Frequency of ESBL encoding genes detected among 623 study isolates

\begin{tabular}{lll}
\hline ESBL type & ESBL encoding gene & $\begin{array}{l}\text { Number } \\
\text { and (\%) of } \\
\text { isolates }\end{array}$ \\
\hline CTX-M & bla $a_{C T X-M 8}$ & $15(32)$ \\
$(N=46.98 \%)$ & $b l a_{C T X-M 15}$ & $9(20)$ \\
& $b l a_{C T X-M 2}$ & $6(13)$ \\
& $b l a_{C T X-M 127}$ & $5(11)$ \\
& $b l a_{C T X-M 14}$ & $3(6)$ \\
& $b l a_{C T X-M 55}$ & $3(6)$ \\
& $b l a_{C T X-M 27}$ & $3(6)$ \\
& $b l a_{C T X-M 65}$ & $1(2)$ \\
& $b l a_{C T X-M 1}$ & $1(2)$ \\
SHV $(\boldsymbol{N}=1.2 \%)$ & $b l a_{S H V-28}$ & $1(2)$ \\
\hline
\end{tabular}

$E S B L$, extended-spectrum beta-lactamase

\section{Discussion}

In the present study, we determined the prevalence of human carriage of antimicrobial-resistant E. coli in a community in Rio de Janeiro city. A small number of subjects were excluded because no $E$. coli was obtained from the rectal specimen, probably related to insufficient yield due to self-sampling.

Resistance frequencies for the top five drugs were high, including SUT and CIP, two of the leading choices for treatment of UTI until recently. The presence of resistant isolates in these high frequencies compromises the microbiota of people in the city. UTI usually starts from an ascending route, with gut microorganisms reaching the bladder [46]. Therefore, one expects a correlation between isolates that colonize the gut and UPEC. In fact, the resistance prevalences among colonizing-isolates of the present study are lower than we observed in a series of 500 UPEC isolates obtained from individuals living in the same region in 2015 [28]. These rates, in turn, are lower than observed around the world among community UTI E. coli isolates, according to the global antimicrobial resistance and the use surveillance system (GLASS) report of WHO [47]. Otherwise, in the present study, we detected ESBL-E from almost $10 \%$ of individuals, similar to the prevalence we observed among UPEC isolates in the city in 2015 (8\%) [28]. However, this prevalence is in the lower side of that reported by many studies in different parts of the world, which vary from 2 to $46 \%$, with the highest levels in countries of West Pacific (46\%) and Asia and Africa (22\%) [4, 48, 49]. The WHO report on third-generation cephalosporin resistance from GLASS estimates a prevalence of almost $60 \%$, including community and hospital isolates, which is much higher than all isolated reports [47].
We observed a worrying trend of increased resistance of gut colonizers over time. Such an upsurge is reported around the world [48, 49] with an annual increasing rate of approximately 5\% [48], which may be related to high levels of antimicrobial consumption in the community, as described by others [5, 48]. In fact, in the present study, the only factor with a significant association with the carriage of MDR-E or ESBL-E was the previous use of antimicrobial agents, as reported by others [50-54]. It would be interesting to reassess such levels late in the year 2021, after the establishment of Brazil as one epicenter of the COVID-19 pandemic, certainly driving a substantial increment of antimicrobial consumption. Traveling abroad was reported only by few subjects, and we found no correlation between other factors such as age, gender, educational level, and comorbid conditions, and carriage of MDR-E or ESBL-E. Antimicrobial use, specifically CIP, was associated with colonization with MDR-E or ESBL-E. The use of a fluoroquinolone has been previously associated with an increased chance of colonization with $E$. coli resistant to the drug [55] and with ESBL producing isolates [56]. This association may be related in part to the frequent presence of quinolone resistance determinants within ESBL gene-carrying plasmids [57].

Several of the E. coli isolates of the present study, including a significant portion of those resistant to CIP, carrying PMQR genes and producing ESBL, belong to phylogenetic groups B2, D/E, or F which comprise more virulent strains, and thus more likely to cause UTI. Indeed, most of such isolates were ExPEC, including among MDR-E and ESBLE. Studies showing that a specific resistant and virulent colonizing strain causes an infection are rare [58]. However, epidemiologic data show an association between risk factors for acquisition of MDR isolates and infection with such microorganisms [52]. The genome plasticity of E. coli and other Enterobacterales is well known and the transfer of genes from gut-colonizers to pathogenic isolates causing infection has been already demonstrated [58]. Therefore, subjects colonized with a virulent strain that has also increased resistance are at higher risk for developing an infection with treatment failure, extended hospital stay, and increased mortality [52].

ESBL produced by isolates were mostly of CTX-M type, mainly CTX-M-8 and CTX-M-15. A bla ${ }_{C T X-M-t y p e}$ gene harboring plasmid usually carries additional genes encoding fluoroquinolone resistance, pAmpC, carbapenemases, and aminoglycoside inactivating enzymes. This way, the use of several antimicrobial agents would give an advantage to isolates with a bla $a_{C T X-M \text {-type }}$ gene [57]. Carbapenemase producers and pAmpC or $\mathrm{mcr}$ gene carriers were not detected in the present population. Resistance to carbapenems has been largely related to hospital-associated infections in Brazil. pAmpC is still a rare 
beta-lactamase, found in less than $1 \%$ of UPEC isolates from community-associated infections in Rio de Janeiro [28].

A limitation of the present study was the use of a swab for collecting fecal specimens, which may have provided a smaller expected yield compared with fecal specimens; resistance rates could have been even higher. In addition, self-collection of specimens might have led to the use of suboptimal sampling procedures. However, we believe that this approach was a critical facilitator making the study feasible. Another limitation of the present study was the lack of strain typing, specially MLST. This information could have led to the description of the presence of pandemic clones as colonizers in the community.

In conclusion, we show that subjects in our community may carry MDR-E and ESBL-E around $10 \%$ of the time. The use of antimicrobial agents, particularly fluoroquinolones [53], is a significant risk factor for the carriage of a resistant isolate, indicating the importance of stewardship programs that must be extensive to outpatients.

Author contribution Káris Maria de Pinho Rodrigues made substantial contributions to the conception or design of the work; analysis, or interpretation of data; drafted the work, approved the version to be published; and agree to be accountable for all aspects of the work in ensuring that questions related to the accuracy or integrity of any part of the work are appropriately investigated and resolved.

Beatriz Meurer Moreira made substantial contributions to the conception or design of the work; drafted the work, revised it critically for important intellectual content, approved the version to be published; and agree to be accountable for all aspects of the work in ensuring that questions related to the accuracy or integrity of any part of the work are appropriately investigated and resolved.

Michelle Pessanha Pinto was responsible for data acquisition, and made substantial contribution to the analysis or interpretation of data, and approved the version to be published.

Danielle Ferreira de Rezende made substantial contribution the analysis and interpretation of data, drafted the work, and approved the version to be published.

João Vitor Almeida Ramalho, Samantha dos Santos Tufic-Garutti, Luís Guilherme de Araújo Longo, Valéria Brígido de Carvalho Girão, Gabriela Caramano de Oliveira, and Lucas Cecílio Vilar made substantial contribution to the analysis and interpretation of data and approved the version to be published.

Funding This study was supported by Coordenação de Aperfeiçoamento de Pessoal de Nível Superior (CAPES)—Finance Code 001, Conselho Nacional de Desenvolvimento Científico e Tecnológico - INPRA - Instituto Nacional de Pesquisa em Resistência Antimicrobiana CNPq 465718/2014-0 and CNPq312205/2019-8 (Characterization of antimicrobial resistance, virulence genes, and population structure of clinical and environmental strains of Escherichia coli obtained in the state of Rio de Janeiro), and Fundação de Amparo à Pesquisa do Estado do Rio de Janeiro, grant no. E-26/211.554/2019 (Programa Rede de Pesquisa em Saúde).

Code availability Not applicable.

Data availability All data can be made available by the authors.

\section{Declarations}

Conflict of interest The authors declare no competing interests.

Ethics approval The study was approved by the Ethics Committee of the University registered at CAAE: 49843315.0.0000.5257.

Consent to participate and consent for publication.

All participants filled an informed consent to participate and to allow the publication of data.

\section{References}

1. Kantele A, Lääveri T, Mero S et al (2015) Antimicrobials increase travelers' risk of colonization by extended-spectrum betalactamase-producing Enterobacteriaceae. Clin Infect Dis 60:837-846

2. Hu Y, Matsui Y, Riley LW (2020) Risk factors for fecal carriage of drug-resistant Escherichia coli: a systematic review and metaanalysis. Antimicrob Resist Infect Control 9:31

3. Haverkate MR, Platteel TN, Fluit AC et al (2017) Quantifying within-household transmission of extended-spectrum $\beta$-lactamaseproducing bacteria. Clin Microbiol Infect 23:46.e1-46.e7

4. Otter JA, Natale A, Batra R et al (2019) Individual- and community-level risk factors for ESBL Enterobacteriaceae colonization identified by universal admission screening in London. Clin Microbiol Infect 25:1259-1265

5. Woerther PL, Burdet C, Chachaty E, Andremont A (2013) Trends in human fecal carriage of extended-spectrum $\beta$-lactamases in the community: toward the globalization of CTX-M. Clin Microbiol Rev 26:744-758

6. Paterson DL (2006) Resistance in gram-negative bacteria: Enterobacteriaceae. Am J Infect Control 34:S20e8 (discussion S64-73)

7. Chong Y, Shimoda S, Shimono N (2018) Current epidemiology, genetic evolution and clinical impact of extended-spectrum $\beta$-lactamase-producing Escherichia coli and Klebsiella pneumoniae. Infect Genet Evol 61:185-188

8. Woerther PL, Angebault C, Jacquier H et al (2011) Massive increase, spread, and exchange of extended spectrum $\beta$-lactamaseencoding genes among intestinal Enterobacteriaceae in hospitalized children with severe acute malnutrition in Niger. Clin Infect Dis 53:677-685

9. Hassuna NA, Khairalla AS, Farahat EM, Hammad AM, AbdelFattah M (2020) Molecular characterization of extended-spectrum $\beta$ lactamase- producing E. coli recovered from communityacquired urinary tract infections in Upper Egypt. Sci Rep. 10:2772

10. Zenati F, Barguigua A, Nayme K et al (2019) Characterization of uropathogenic ESBL-producing Escherichia coli isolated from hospitalized patients in western Algeria. J Infect Dev Ctries 13:291-302

11. Otter JA, Mutters NT, Tacconelli E, Gikas A, Holmes AH (2015) Controversies in guidelines for the control of multidrug-resistant Gram-negative bacteria in EU countries. Clin Microbiol Infect. 21:1057e66

12. Tacconelli E, Cataldo MA, Dancer SJ et al (2014) European Society of Clinical Microbiology. ESCMID guidelines for the management of the infection control measures to reduce transmission of multidrug-resistant Gram-negative bacteria in hospitalized patients. Clin Microbiol Infect 1:1-55

13. Poirel L, Madec JY, Lupo A et al. (2018) Antimicrobial Resistance in Escherichia coli. Microbiol Spectr 6(4). https://doi.org/10.1128/ microbiolspec.ARBA-0026-2017 
14. Boyd EF, Hartl DL (1998) Chromosomal regions specific to pathogenic isolates of Escherichia coli have a phylogenetically clustered distribution. J Bacteriol 180:1159-1165

15. Smith JL, Fratamico PM, Gunther NW (2007) Extraintestinal pathogenic Escherichia coli. Foodborne Pathog Dis 4:134-163

16. Johnson JR, Russo TA (2005) Molecular epidemiology of extraintestinal pathogenic (uropathogenic) Escherichia coli. Int J Med Microbiol 295:383-404

17. O’Neill J (2016) Tackling drug resistant infections globally: final report and recommendations. Review on antimicrobial resistance. Available at https://amr-review.org/sites/default/files/160518_ Final\%20paper_with\%20cover.pdf. Accessed 6 Jan 2022.

18. Ministério da Saúde (2020) Orientações do Ministério da Saúde para manuseio medicamentoso precoce de pacientes com diagnóstico da COVID-19. Available at https://www.gov.br/saude/pt-br/ centrais-de-conteudo/arquivos/orientacoes-manuseiomedicam entoso-covid19-pdf. Accessed 6 Jan 2022.

19. Lee JH, Subhadra B, Son YJ et al (2016) Phylogenetic group distributions, virulence factors and antimicrobial resistance properties of uropathogenic Escherichia coli strains isolated from patients with urinary tract infections in South Korea. Lett Appl Microbiol 62:84-90

20. Giray B, Uçar FB, Aydemir SS (2012) Characterization of uropathogenic Escherichia coli strains obtained from urology outpatient clinic of Ege medical faculty in Izmir. Turk J Med Sci 42:1328-1337

21. Riley LW (2014) Pandemic lineages of extraintestinal pathogenic Escherichia coli. Clin Microbiol Infect 20:380-390

22. Denamur E, Clermont O, Bonacorsi S, Gordon D (2021) The population genetics of pathogenic Escherichia coli. Nat Rev Microbiol 19(1):37-54

23. Brisola MC, Crecencio RB, Bitner DS et al (2019) Escherichia coli used as a biomarker of antimicrobial resistance in pig farms of Southern Brazil. Sci Total Environ 647:362-368

24. Lentz SAM, Adam FC, Rivas PM et al (2020) High levels of resistance to cephalosporins associated with the presence of extended-spectrum and AmpC $\beta$-lactamases in Escherichia coli from Broilers in Southern Brazil. Microb Drug Resist 26:531-535

25. Botelho LAB, Kraychete GB, Rocha PB et al (2020) CTX-Mand $\mathrm{pAmpC}$-encoding genes are associated with similar mobile genetic elements in Escherichia coli isolated from different brands of Brazilian chicken meat. Microb Drug Resist 26:14-20

26. Batalha de Jesus AA, Freitas AAR, de Souza JC et al (2019) Highlevel multidrug-resistant Escherichia coli isolates from wild birds in a large urban environment. Microb Drug Resist 25:167-172

27. Fernandes MR, Sellera FP, Moura Q, Esposito F, Sabino CP, Lincopan N (2020) Identification and genomic features of halotolerant extended-spectrum- $\beta$-lactamase (CTX-M)-producing Escherichia coli in urban-impacted coastal waters. Southeast Brazil. Mar Pollut Bull. 150:110689

28. da-Silva APS, Sousa VS, Longo LGA et al (2020) Prevalence of fluoroquinolone-resistant and broad-spectrum cephalosporinresistant community-acquired urinary tract infections in Rio de Janeiro: impact of Escherichia coli genotypes ST69 and ST131. Infect Genet Evol. 85:104452. https://doi.org/10.1016/j.meegid. 2020.104452

29. Tufic-Garutti SS, Ramalho JVA, Longo LGA et al (2021) Acquisition of antimicrobial resistance determinants in Enterobacterales by international travelers from a large urban setting in Brazil. Trav Med Infect Dis 41:102028. https://doi.org/10.1016/j.tmaid.2021. 102028

30. Jarlier V, Nicolas MH, Fournier G, Philippon A (1988) Extended broad-spectrum beta-lactamases conferring transferable resistance to newer beta-lactam agents in Enterobacteriaceae: hospital prevalence and susceptibility patterns. Rev Infect Dis 10:867-878
31. Magiorakos AP, Srinivasan A, Carey RB et al (2012) Multidrugresistant, extensively drug-resistant and pandrug-resistant bacteria: an international expert proposal for interim standard definitions for acquired resistance. Clin Microbiol Infect 18:268-281

32. Wang XR, Chen JC, Kang Y, Jiang N, An SC, Gao ZC (2012) Prevalence and characterization of plasmid-mediated blaESBL with their genetic environment in Escherichia coli and Klebsiella pneumoniae in patients with pneumonia. Chin Med J (Engl) 125:894-900

33. Pérez-Pérez FJ, Hanson ND (2002) Detection of plasmid-mediated AmpC $\beta$-lactamase genes in clinical isolates by using multiplex PCR. J Clin Microbiol 40:2153-2162

34. Rebelo AR, Bortolaia V, Kjeldgaard JS et al. Malhotra-Kumar, L. Villa, A. Carattoli and R. S. Hendriksen (2018) Multiplex PCR for detection of plasmid-mediated colistin resistance determinants, mcr- 1 , mcr-2, mcr-3, mcr-4 and mcr-5 for surveillance purposes. Eurosurveillance. 23(6)

35. Kraychete GB, Botelho LAB, Campana EH et al (2016) Updated multiplex PCR for detection of all six plasmidmediated qnr gene families. Antimicrob Agents Chemother 60(12):7524-7526

36. Cattoir V, Poirel L, Rotimi V et al (2007) Multiplex PCR for detection of plasmid-mediated quinolone resistance qnr genes in ESBL-producing enterobacterial isolates. J Antimicrob Chemother 60(2):394-397. https://doi.org/10.1093/jac/dkm204

37. Park CH, Robicsek A, Jacoby GA, Sahm D, Hooper DC (2006) Prevalence in the United States of $a a c\left(6^{\prime}\right)-I b-c r$ encoding a ciprofloxacin-modifying enzyme. Antimicrob Agents Chemother 50:3953-3955

38. Yamane K, Wachino J, Suzuki S, Arakawa Y (2008) Plasmidmediated qepA gene among Escherichia coli clinical isolates from Japan. Antimicrob Agents Chemother 52(4):1564-1566. https:// doi.org/10.1128/AAC.01137-07

39. Clermont O, Dhanji H, Upton M et al (2009) Rapid detection of the O25b-ST131 clone of Escherichia coli encompassing the CTX-M-15-producing strains. J Antimicrob Chemother 64:274-277

40. Clermont O, Bonacorsi S, Bingen E (2000) Rapid and simple determination of the Escherichia coli phylogenetic group. Appl Environ Microbiol 66:4555-4558

41. Clermont O, Christenson JK, Denamur E (2013) Gordon DM (2013) The clermont Escherichia coli phylo-typing method revisited: improvement of specificity and detection of new phylogroups. Environ Microbiol Rep 5(1):58-65

42. Johnson JR (2003) Microbial virulence determinants and the pathogenesis of urinary tract infection. Infect Dis Clin North Am $17: 261-278$

43. Le Bouguenec C, Archambaud M, Labigne A (1992) Rapid and specific detection of the pap, afa, and sfa adhesin-encoding operons in uropathogenic Escherichia coli strains by polymerase chain reaction. J Clin Microbiol 30:1189-1193

44. Johnson JR, Brown JJ (1996) A novel multiply-primed polymerase chain reaction assay for identification of variant papG genes encoding the Gal(al-4)Galbinding PapG adhesins of Escherichia coli. J Infect Dis 173:920-926

45. Johnson JR, Stell AL (2000) Extended virulence genotypes of Escherichia coli strains from patients with urosepsis in relation to phylogeny and host compromise. J Infect Dis 181:261e72

46. Flores-Mireles AL, Walker JN, Caparon M, Hultgren SJ (2015) Urinary tract infections: epidemiology, mechanisms of infection and treatment options. Nat Rev Microbiol 13:269-284

47. Global antimicrobial resistance and use surveillance system (GLASS) report (2021) Geneva: World Health Organization

48. Karanika S, Karantanos T, Arvanitis M, Grigoras C, Mylonakis E (2016) Fecal colonization with extended-spectrum betalactamase-producing enterobacteriaceae and risk factors among 
healthy individuals: a systematic review and metaanalysis. Clin Infect Dis 63:310-318

49. Pires J, Kuenzli E, Hauser C et al (2018) Intestinal colonisation with extended-spectrum cephalosporin-resistant Enterobacteriaceae in different populations in Switzerland: prevalence, risk factors and molecular features. J Glob Antimicrob Resist 12:17-19

50. Huijbers PM, de Kraker M, Graat EA et al (2013) Prevalence of extended-spectrum $\beta$-lactamase-producing Enterobacteriaceae in humans living in municipalities with high and low broiler density. Clin Microbiol Infect 19:E256-E259

51. Nakai H, Hagihara M, Kato H et al (2016) Prevalence and risk factors of infections caused by extended-spectrum $\beta$-lactamase (ESBL)-producing Enterobacteriaceae. J Infect Chemother 22:319-326

52. Woerther PL, Andremont A, Kantele A (2017) Travel-acquired ESBL-producing Enterobacteriaceae: impact of colonization at individual and community level. J Travel Med 24:S29-S34

53. Kantele A, Mero S, Kirveskari J, Lääveri T (2017) Fluoroquinolone antibiotic users select fluoroquinolone-resistant ESBLproducing Enterobacteriaceae (ESBL-PE) - Data of a prospective traveler study. Travel Med Infect Dis 16:23-30

54. Arcilla MS, Van Hattem JM, Bootsma MCJ et al (2019) Prevalence and risk factors for carriage of ESBL-producing Enterobacteriaceae in a population of Dutch travelers: A cross-sectional study. Travel Med Infect Dis 33:101547. https://doi.org/10.1016/j. tmaid.2019.101547

55. Hsueh PR, Chen WH, Luh KT (2005) Relationships between antimicrobial use and antimicrobial resistance in Gram-negative bacteria causing nosocomial infections from 1991-2003 at a university hospital in Taiwan. Int J Antimicrob Agents 26:463-472

56. Reuland EA, Sonder GJ, Stolte I et al (2016) Travel to Asia and traveller's diarrhoea with antibiotic treatment are independent risk factors for acquiring ciprofloxacin-resistant and extended spectrum $\beta$-lactamase-producing Enterobacteriaceae-a prospective cohort study. Clin Microbiol Infect 2:731.e1-7

57. Cantón R, González-Alba JM, Galán JC (2012) CTX-M enzymes: origin and diffusion. Front Microbiol 3:110

58. Porse A, Gumpert H, Kubicek-Sutherland JZ et al (2017) Genome dynamics of Escherichia coli during antibiotic treatment: transfer, loss, and persistence of genetic elements in situ of the infant gut. Front Cell Infect Microbiol 12(7):126. https://doi.org/10.3389/ fcimb.2017.00126.eCollection2017

Publisher's Note Springer Nature remains neutral with regard to jurisdictional claims in published maps and institutional affiliations. 